\title{
Effects of functional polysaccharide from silkworm as an immunostimulant on transcriptional profiling and disease resistance in fish
}

\author{
M.F.Z. Ali ${ }^{1}$, S. Nakahara ${ }^{1}$, Y. Otsu ${ }^{1}$, A. Ido ${ }^{1}$, C. Miura ${ }^{1,2}$ and T. Miura $^{1 *}$ iD \\ ${ }^{1}$ Graduate School of Agriculture, Ehime University, 3-5-7, Tarumi, Matsuyama, Ehime 790-8566, Japan; ${ }^{2}$ Department of \\ Global Environment Studies, Faculty of Environmental Studies, Hiroshima Institute of Technology, 2-1-1, Miyake, Saeki-ku, \\ Hiroshima 731-5193, Japan; miutake@agr.ehime-u.ac.jp
}

Received: 22 June 2021 / Accepted: 26 August 2021

(c) 2021 Wageningen Academic Publishers

OPEN ACCESS CC (i) RESEARCH ARTICLE

\begin{abstract}
Dietary manipulation to maintain fish health and reduce bacterial infection through the use of immunostimulants has been widely used worldwide. A broad range of bioactive substances capable of optimising animal health has been found in several insect species, including antimicrobial/antiviral peptides, polysaccharides such as chitin, lauric acid, and insect products such as honey. Recently, we identified a novel bioactive polysaccharide from Bombyx mori, termed silkrose-BM, that can activate innate immunity in mammalian RAW264.7 macrophages and provide effective protection against vibriosis in penaeid prawns. However, the efficacy of dietary silkrose-BM in teleosts remains unclear. Here, we investigated the effects of dietary inclusion of silkrose-BM in Japanese medaka (Oryzias latipes) after they were artificially challenged with Edwardsiella tarda. The survival of medaka after infection with $E$. tarda was significantly improved by dietary silkrose-BM at a concentration of 10, 100, and $1000 \mathrm{ng} / \mathrm{g}$. RNA-seq analysis was performed in the intestine and liver of the medaka to identify changes in the transcriptional profiling evoked by silkrose-BM. The dietary silkrose-BM group showed 1,194 and 2,259 differentially expressed genes (DEGs) in the intestine and liver, respectively, when compared with the control group prior to $E$. tarda infection. Functional enrichment analysis of DEGs showed several putative genes involved in the Toll-like receptor/nuclear factor $\mathrm{kB}$ pathway, cytokine-cytokine receptor interactions, complement cascade, antimicrobial peptides, and junctional modification. Taken together, these results suggest that silkrose-BM used as an immunostimulant can improve the immune system and resistance to edwardsiellosis in teleosts.
\end{abstract}

Keywords: polysaccharide, insect, immunostimulant, Japanese medaka, RNA-seq, immune response

\section{Introduction}

In recent years, insects have attracted considerable interest worldwide due to being not only a promising protein source for animal feed production (Makkar et al., 2014; Van Huis, 2021) but also as organisms that can produce a variety of useful substances capable of optimising animal health (Chernysh et al., 2002; Gasco et al., 2018; Ratcliffe et al., 2014). Dietary inclusion of insect meal, such as housefly (Musca domestica), yellow mealworm (Tenebrio molitor), and black soldier fly (Hermetia illucens), has been widely used in various commercial fish and shellfish species with notable success in modulating immune responses and enhancing resistance to bacterial infection (Chaklader et al., 2019; Henry et al., 2018; Ido et al., 2015, 2019; Motte et al., 2019; Xiao et al., 2018). We recently discovered novel bioactive polysaccharides, termed dipterose-BC (Ohta et al., 2014), dipterose-BSF (Ali et al., 2019), silkrose-AY (Ohta et al., 2015), and silkrose-BM (Ali et al., 2018), in Bactrocera cucurbitae larvae, $H$. illucens larvae, Antheraea yamamai pupae, and Bombyx mori pupae, respectively. These insect-derived bioactive polysaccharides can effectively induce nitric oxide production and the expression of proinflammatory cytokines and interferon $\beta$ via the Tolllike receptor (TLR)/nuclear factor $\kappa \mathrm{B}(\mathrm{NF}-\mathrm{kB})$ pathway in mouse RAW264.7 macrophages, indicating further potential benefits as functional feed ingredients for agriculture and aquaculture. 
Edwardsiella tarda, an opportunistic gram-negative bacterium, is a major pathogen that represents a serious economic threat to the aquaculture industry. E. tarda infection can induce a serious systematic illness, called edwardsiellosis, in a broad range of freshwater and marine fish species (Clavijo et al., 2002; Matsuyama et al., 2005; Meyer and Bullock, 1973; Padrós et al., 2006). Clinical manifestations of edwardsiellosis include haemorrhagic septicaemia and abscess formation on the body surface and internal organs of an infected host; the condition is often associated with environmental stresses such as poor water quality, temperature changes, and high organic content (Mohanty and Sahoo, 2007). Numerous ways to develop effective protection strategies against edwardsiellosis have been extensively studied, including the use of vaccines to improve host immunity (Castro et al., 2008; Park et al., 2012) and the application of antibiotic agents (Mohanty and Sahoo, 2007). Although many attenuated vaccines have been developed using various methods and have been shown to be highly effective in laboratory trials, vaccination as a general practice for the prevention of edwardsiellosis remains limited, mainly due to its high costs, minimal effectiveness, labour intensiveness, and failure to meet producers' expectations (Bebak and Wagner, 2012; Park et al., 2012). However, increasing concerns regarding the problems associated with the use of large quantities of antibiotics in aquaculture prompted us to search for safer and more efficient techniques for controlling diseases in aquaculture (Cabello, 2006). The use of immunomodulatory substances, such as dipterose and silkrose, is increasingly being suggested as a novel strategy to protect against edwardsiellosis.

Rapid advances in molecular technologies have resulted in a new method for gene expression profiling, known as RNA sequencing (RNA-seq). Recently, this technology has been widely applied to obtain a comprehensive understanding of the molecular mechanisms involved in fish immune systems as well as in tissue-specific immunoregulatory responses to immunostimulant-containing diets (Martin et al., 2016; Salinas and Magadán, 2017; Ye et al., 2018). Bioactive insectderived polysaccharides are relatively novel substances and their molecular effects in fish remain undefined; therefore, utilising high-throughput gene expression approaches such as RNA-seq could provide better insights into the interactions between host molecular systems and bioactive insect-derived polysaccharides. Previously, we conducted gene expression profiling by microarray analysis of Japanese medaka (Oryzias latipes) fed with silkrose from A. yamamai (silkrose-AY) and revealed the up-regulation of several putative genes involved in the fish innate immune system and epithelial junctional modification (Ali et al., 2021).

Here, we examined the efficacy of a novel immunostimulatory polysaccharide derived from silkmoth (B. mori), termed silkrose-BM, against E. tarda in a model organism of teleosts, the Japanese medaka (O. latipes). To begin to understand the underlying mechanism of dietary silkrose$\mathrm{BM}$ at the transcriptional level, we conducted transcriptome profiling of the intestine and liver of medaka using RNA-seq technology. The identification of molecular mechanisms in these two important tissues for the fish immune system will advance our knowledge of the immunostimulatory effect in teleosts resulting from the dietary inclusion of silkrose-BM.

\section{Material and methods}

\section{Purification of silkrose-BM}

Silkrose from B. mori (silkrose-BM) was purified as previously described (Ali et al., 2018; Ohta et al., 2015). B. mori pupae from Ozu (3332'46.3'N $132^{\circ} 32^{\prime} 56.1^{\prime} \mathrm{E}$; Ehime Prefecture, Japan) were provided by United Silk Co., Ltd (Matsuyama, Ehime, Japan). Briefly, crude polysaccharides obtained from dried powdered $B$. mori pupae by a successive series of water extraction and ethanol precipitation steps were fractionised and purified by gel filtration and anion-exchange chromatography on a fast protein liquid chromatography (FPLC) system. A HiPrep 26/60 Sephacryl S-500HR column and a HiPrep Q XL 16/10 column (GE Healthcare, Chicago, IL, USA) were used for gel filtration and anion-exchange chromatography, respectively. The positive fractions in the nitric oxide production assay were pooled and enriched by the addition of four volumes of $100 \%(\mathrm{v} / \mathrm{v})$ ethanol and stored overnight at $4{ }^{\circ} \mathrm{C}$. The precipitate was collected by centrifugation then lyophilised to obtain the bioactive polysaccharide. The homogeneity of the purified polysaccharides was then determined using a high-performance liquid chromatography system (HPLC; Hitachi, Tokyo, Japan) equipped with a Showdex SB-807 HQ size-exclusion chromatographic column (Showa Denko K.K., Minato, Japan) and a refractive index detector. The polysaccharide was detected as a single symmetrical peak, indicating the homogeneity of the substance (Ohta et al., 2015). The phenol-sulfuric acid method (Dubois et al., 1956) with glucose as a standard was used to determine the total carbohydrate levels of the purified polysaccharides.

\section{Fish rearing}

All animal procedures were performed under the guidelines of the Regulations for Animal Experiments approved by the Institutional Animal Care and Use Committee (IACUC) of Ehime University (Permit Number: 3908). Three-month-old Japanese medaka (O. latipes), with a weight and fork-length range between $250-350 \mathrm{mg}$ and $30-35 \mathrm{~mm}$, respectively, were used for all experiments. The animals were maintained in fresh water at a temperature of $25 \pm 2{ }^{\circ} \mathrm{C}$. The daily cycle for the animals was $14 \mathrm{~h}$ light and $10 \mathrm{~h}$ dark. All body measurements and surgical manipulations were conducted under the effect of 2-phenoxyethanol at the concentration 
of 0.01 and $0.2 \%$, respectively, and all efforts were made to minimise suffering.

\section{Preparation of experimental diets}

The experimental diets were prepared using a previously described method (Ali et al., 2021). The composition of the basal diets for the medaka is shown in Table S1. Briefly, dry ingredients were well mixed, supplemented with fish oil, and finally mixed with water before pelletisation using a cylindrical granulator, ABV-120L (Akira Kiko, Fukui, Japan). Following granulation, the pellets were completely air-dried at $60{ }^{\circ} \mathrm{C}$ for $1-2$ days. Experimental diets were prepared by adding final concentrations of silkrose-BM of $10 \mathrm{ng} / \mathrm{g}$, $100 \mathrm{ng} / \mathrm{g}$, or $1000 \mathrm{ng} / \mathrm{g}$ to the base diet ingredients. No ingredients were substituted when adding silkrose-BM, as the supplemented quantities were very small $(\leq 1000 \mathrm{ng} / \mathrm{g})$, and the nutritional influence of silkrose-BM was considered negligible.

\section{Experimental methods and sample collection}

Fish ( $\mathrm{n}=18$ per tank) were fed to satiation (three times per day at $8 \mathrm{am}, 12 \mathrm{pm}$, and $4 \mathrm{pm}$ ) with one of the four types of diets (feed containing $0,10,100$, or $1000 \mathrm{ng} / \mathrm{g}$ of silkroseBM) for two weeks. Triplicate tanks (45 l) were used for each type of diet. Two weeks following the feeding regime, six fish per treatment group ( $n=2$ per tank; $n=6$ per treatment group) were randomly captured for tissue collection. After being euthanised with 2-phenoxyethanol (0.2\%), the entire intestine and liver of each fish was removed and immediately stored in RNAlater solution (Invitrogen, Waltham, MA, USA) at $-20^{\circ} \mathrm{C}$ until further processing.

\section{Preparation of $E$. tarda and challenge tests}

The pathogen was isolated from the kidneys of $E$. tardainfected red sea bream (Pagrus major) cultured in Ainan, Ehime Prefecture, Japan. E. tarda was inoculated in Salmonella-Shigella medium (Nihon Pharmaceutical, Tokyo, Japan) and incubated for $48 \mathrm{~h}$ at $28^{\circ} \mathrm{C}$. Bacterial colonies were collected and cultured on sterilised Luria-Bertani medium (LB; $10 \mathrm{~g}$ tryptone, $5 \mathrm{~g}$ yeast extract, $5 \mathrm{~g} / \mathrm{l} \mathrm{NaCl}$ ) for $24 \mathrm{~h}$ with vigorous agitation $(200 \mathrm{rpm} / \mathrm{min})$ at $28^{\circ} \mathrm{C}$. The bacteria were harvested by centrifugation $(6,000 \mathrm{rpm})$ for 5 $\min$ at $4{ }^{\circ} \mathrm{C}$. Following centrifugation, the supernatant was removed and the precipitated bacteria were washed twice with $50 \mathrm{ml}$ sterilised phosphate-buffered saline (PBS; $8 \mathrm{~g}$ $\left.\mathrm{NaCl}, 0.2 \mathrm{~g} \mathrm{KCl}, 1.15 \mathrm{~g} \mathrm{Na}_{2} \mathrm{HPO}_{4}, 0.2 \mathrm{~g} \mathrm{KH}_{2} \mathrm{PO}_{4}, \mathrm{pH} 7.4\right)$. Finally, the precipitated bacteria were diluted in sterilised PBS to adjust the bacterial titer to $1 \times 10^{7}$ colony-forming units $(\mathrm{cfu}) / \mathrm{ml}$. The concentration of the bacteria in cfu per $\mathrm{ml}$ was determined by serially plating $10 \mu \mathrm{l}$ of the bacterial culture in 10-fold serial dilutions onto tryptone-soya agar (TSA; Nissui Pharmaceutical, Tokyo, Japan) and culturing at $28^{\circ} \mathrm{C}$ for 24 hours.
Groups of fish fed on one of the four types of diets (feed containing $0,10,100$, or $1000 \mathrm{ng} / \mathrm{g}$ silkrose-BM) were used for the challenge test. Triplicate tanks per treatment group with 16 fish/tank were used. After 2 weeks of the feeding regime, fish were exposed to the bacterial suspension of $E$. tarda $\left(1 \times 10^{7} \mathrm{cfu} / \mathrm{ml}\right)$ by static immersion for $5 \mathrm{~h}$. Following immersion, the fish were moved into $45-1$ tanks equipped with an underwater air-filtration system and maintained for 20 days. The fish were continuously fed with the experimental diet following the challenge test until the end of the observation period. Fish were observed daily for signs of disease and mortality. Any dead fish were routinely removed. The survival rate was determined at 3 days after deaths in the basal diet groups had stopped.

\section{Total RNA isolation and RNA-seq analysis}

RNA-seq analysis was performed in two dietary treatment groups: the $1000 \mathrm{ng} / \mathrm{g}$ silkrose-BM-containing diet and basal diet groups. For comparison purposes, the samples collected from fish fed on the $1000 \mathrm{ng} / \mathrm{g}$ silkrose-BM-containing diet and the base diet were named S-N and C-N, respectively. Total RNA was purified from the medaka whole intestine and liver using an RNeasy Universal Mini Kit (Qiagen, Hilden, Germany), following the manufacturer's protocol. The total RNA yield was assessed spectrophotometrically using a Nanophotometer P330 (Implen, München, Germany). RNA from six individuals sampled in each group were pooled into one $1.5 \mathrm{ml}$ Eppendorf tube to equalise the amount of total RNA. The volume after pooling was adjusted to be more than $20 \mu \mathrm{l}$ and total RNA concentration to be more than $50 \mathrm{ng} / \mu \mathrm{l}$. Prior to the preparation of library DNA, the concentration of pooled total RNA was measured using a QubitRNA HS Assay Kit (Thermo Fisher Scientific, Waltham, MA, USA), and the quality of the samples was confirmed using a Qsep100 DNA Fragment Analyzer and an RNA R1 Cartridge (BiOptic, New Taipei District, Taiwan). Library DNA was prepared using a KAPA Stranded mRNAseq kit (KAPA BIO SYSTEMS, Wilmington, MA, USA), according to the manufacturer's instructions. The prepared library DNA solution was subjected to concentration measurements using Qubit and dsDNA HS Assay Kits (Thermo Fisher Scientific), and quality confirmation of library DNA was performed using a Fragment Analyzer and a dsDNA 915 Reagent Kit (Advanced Analytical Technologies, Ankeny, IA, USA).

Library DNA was cyclised using a MGIEeasy Circularization Kit (MGI Tech Co., Ltd, Yantian District, Shenzhen, China, P.R.) and then prepared as DNA nanoballs (DNBs) using a DNBSEQ-G400RS High-throughput Sequencing Kit (MGI Tech Co., Ltd), according to the manufacturer's instructions. Sequences of DNBs were read using a NextSeq 500 (Illumina, San Diego, Ca, USA) under the condition of $2 \times 76 \mathrm{bp}$. 
After removing adapter sequences from the obtained sequence data using Cutadapt (ver. 1.9.1, Martin, 2011), bases with a quality score of less than 20 and paired reads with less than 30 bases were removed using Sickle (ver. 1.33). Using HISAT2 (ver. 2.2.0, Kim et al., 2019), the filtered reads were mapped to the reference sequence of O. latipes (https:// www.ncbi.nlm.nih.gov/assembly/GCF_002234675.1/) using the SAM format as the data output. The SAM format was converted into BAM format using Samtools (ver. 1.10, Li et $a l ., 2009)$, and then indexing was performed. The reads that mapped to the gene regions of the reference sequence were counted using featureCounts (ver. 2.0.0, Liao et al., 2014). Corrections for the total number of reads and gene length between samples were performed using reads per kilobase million (RPKM) normalisation. Differentially expressed genes (DEGs) were identified using TMM-edgeR-TMM pipeline after normalisation using the DEGES normalisation method in TCC (ver.1.26.0, Sun et al., 2013). Log2 foldchanges in genes of $\geq 1$ or $\leq-1$ times with $P<0.05$ when compared with the $\mathrm{C}-\mathrm{N}$ group were set as the thresholds for up- and down-regulation, respectively.

Gene Ontology (GO) enrichment and pathway enrichment analysis of the DEGs were performed using ClusterProfiler (ver. 3.18.1, Yu et al., 2012) within the BioConductor package in R software (R Core Team, 2020). The Kyoto Encyclopaedia of Genes and Genomes (KEGG) database was used for pathway enrichment analysis (https://www. kegg.jp/).

\section{Confirmation of RNA-seq results by quantitative RT-PCR}

Seven genes were selected to confirm the RNA-seq (quantification) data by quantitative reverse-transcribed PCR (qRT-PCR) using a SsoFast EvaGreen Supermix (Bio-Rad Laboratories, Hercules, CA, USA), according to the manufacturer's instructions. The same RNA sample sets ( $\mathrm{n}=6$ per treatment group; control and silkrose-BMcontaining diet groups) were used for both RNA-seq analysis and the qRT-PCR confirmation assay. First-strand cDNA was obtained from $500 \mu \mathrm{g}$ of total RNA using a QuantiTect Reverse Transcription Kit (Qiagen). The genespecific primers used for qRT-PCR are listed in Table S2 and Figure S7. Melting curve analyses were performed following amplification for each gene to ensure only a single product had been amplified. The thermocycling process was performed in a Multiplate PCR Plates 96-well white plate (Bio-Rad Laboratories) using a quantitative RT-PCR detection system (Bio-Rad Laboratories) with the following conditions: $95^{\circ} \mathrm{C}$ for $30 \mathrm{~s}$, then 40 cycles of $95^{\circ} \mathrm{C}$ for $5 \mathrm{~s}$ and $55^{\circ} \mathrm{C}$ for $5 \mathrm{~s}$. The relative gene expression was calculated using the comparative threshold (CT) cycle method described by Livak and Schmittgen (2001), with $\beta$-actin as an endogenous reference. $\beta$-actin was chosen as an endogenous control gene for normalisation due to its invariant expression across experimental treatments in medaka (Ali et al., 2021). No significant differences were observed from the $\beta$-actin's $2^{-\Delta \mathrm{Ct}}$ values between control and silkrose-BM group in the liver $(P=0.090)$ and intestine $(P=0.4419)$ of medaka (Figure S8).

\section{Calculation and statistical analysis}

Data are expressed as means \pm standard error. Statistical analysis of survival rates in the challenge test was conducted using the Kaplan-Meier survival estimator followed by a log-rank (Mantel-Cox) test at the 0.05 level of significance for the comparison analysis. For confirmation of RNAseq results by qRT-PCR, a Spearman's correlation test was performed to statistically compare the $\log 2$ foldchange from qRT-PCR with the RNA-seq results. Other measurement items were explored using a Student's $t$-test with significance set at $P<0.05$. All tests were conducted using R software.

\section{Results}

\section{Dietary effects of silkrose-BM on protection from $E$. tarda in Japanese medaka}

To evaluate whether dietary supplementation with silkroseBM had any effects on the resistance of teleosts to bacterial pathogens, we performed a challenge test using Japanese medaka (O. latipes), a teleost model organism, against a gram-negative bacterium, $E$. tarda. Two weeks following exposure to $E$. tarda, fish in the silkrose-BM-containing diet groups showed a much higher survival rate than those in the control group (Figure 1). In addition, a dose-dependent increase in the survival of the medaka was observed in the silkrose-BM-containing diet groups. The average survival rates on post-challenge day 14 were $4.17 \%(1 / 16 ; 1 / 16$; 0/16 fish), 60.42\% (10/16; 9/16; 10/16 fish), 66.67\% (11/16; $10 / 16 ; 11 / 16$ fish), and $83.33 \%$ (13/16; 12/16; $15 / 16$ fish) in the control, 10, 100, and $1000 \mathrm{ng} / \mathrm{g}$ silkrose-BM groups, respectively. The survival rates from day 14 post-infection until the end of the observation period at day 20 postinfection remained the same.

\section{Analysis of transcriptome changes by RNA-seq in response to dietary silkrose-BM}

To explore the transcriptome changes in teleosts caused by dietary silkrose-BM, we performed RNA-seq analysis of intestine and liver tissues isolated from medaka that had been fed with basal diet (C-N) or $1000 \mathrm{ng} / \mathrm{g}$ silkrose-BMcontaining diet (S-N) for two weeks. RNA-seq generated $14,130,208 \pm 1,524,750.81$ raw reads. After removing low-quality reads and adapter sequences, we obtained $13,430,341 \pm 1,383,473.59$ clean reads, accounting for 93.96 to $96.05 \%$ of raw reads (Table S3). Quality control analysis indicated the values of Q20 and Q30 were more than 91.5 and $88.0 \%$, respectively. The resulting clean reads 


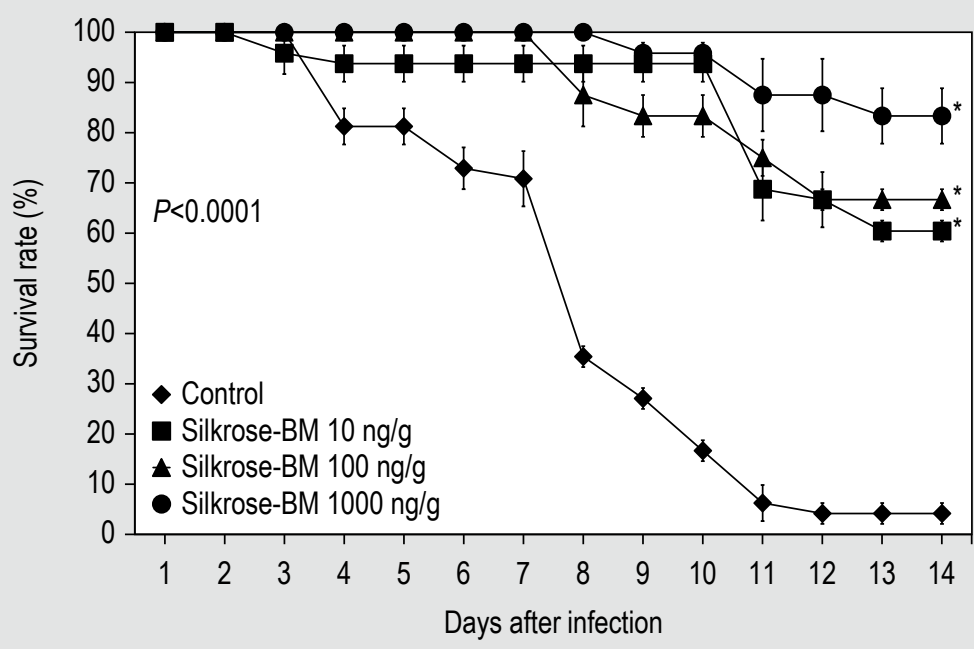

Figure 1. Survival rate of medaka after immersion with Edwardsiella tarda strain $A$ (input dose: $1 \times 10^{7} \mathrm{cfu} / \mathrm{ml}$ ). Triplicate tanks with 16 individual fish ( $n=16 /$ tank; n=48/study group) per study groups was used. Control diet group (solid diamond), $10 \mathrm{ng} / \mathrm{g}$ silkrose-Bombyx mori (BM) diet group (solid square), $100 \mathrm{ng} / \mathrm{g}$ silkrose-BM diet group (solid triangle), and $1000 \mathrm{ng} / \mathrm{g}$ silkrose-BM diet group (solid square). The mortality was recorded for 20 days after the bacterial challenge and the survival rates from day 14 to 20 post-infection were remained the same. Asterisks indicate statistically significant differences compared with the control group by the Kaplan-Meier log-rank test at an $\alpha$-level of 0.05 (log-rank $P<0.05$ ). Each bar indicates the standard error of the means from three replicates.

were then mapped to the reference sequence of $O$. latipes (GCF_002234675.1) using HISAT2 (ver. 2.2.0) for each sample, a total of $11,168,262 \pm 1,182,118.83$ clean reads was aligned, yielding an average successful mapping rate of $83.15 \%$ (Table S4).

After assembling and calculating the transcripts using RPKM normalisation, a total of 51,784 transcripts were detected in assembled transcripts, of which $37,410,37,844$, 44,244 , and 43,830 transcripts were identified as expressed in C-N liver, S-N liver, C-N intestine, and S-N intestine, respectively, accounting for $72.24,73.08,85.44$, and $84.64 \%$ of expressed transcripts, respectively (Figure S1a).

\section{Identification of DEGs induced by dietary silkrose-BM}

DEGs were identified using a threshold of $\mid \log 2$ foldchange $\mid \geq 1$ and $P$-value $<0.05$. Differential expression analysis using the TCC package (ver.1.26.0) showed that 1,194 genes in the intestine of medaka were differentially expressed between the S-N and C-N groups, including 659 up-regulated genes and 535 down-regulated genes. Using the same threshold, 2,259 DEGs were observed in the liver of medaka when comparing S-N and C-N groups, which included 1,026 up-regulated genes and 1,233 down-regulated genes. To visualise the distribution of up-regulated and down-regulated genes in the intestine and liver of medaka, the resulting DEGs were plotted into volcano plot using the ggplot2 package (ver. 3.3.3), as shown in Figure S1b-c.

\section{Gene ontology enrichment results of DEGs}

GO enrichment analysis was used to determine the molecular mechanism underlying the dietary effect of silkrose-BM in the intestine and liver of medaka. DEGs from the comparison between $\mathrm{S}-\mathrm{N}$ and $\mathrm{C}-\mathrm{N}$ groups were entered into the software package ClusterProfiler for over-representation analysis (ORA). The analysis showed $105 \mathrm{GO}$ terms of biological processes and 5 of molecular functions were significantly enriched $(P$-adjust $<0.05)$ in the intestine of medaka after they were fed on a diet that included silkrose-BM (Figure S2a-b and Table S5). The resulting GO terms of biological processes in the intestine were further analysed using REVIGO (Supek et al., 2011), to find representative subgroups of the terms using semantic similarity measures (Figure S3). Among them, the representative terms included muscle contraction (GO:0006936), ion transmembrane transport (GO:0034220), fertilisation and reproduction (GO:0009566; GO:0000003), actomyosin structure organisation (GO:0031032), positive regulation of acrosome (GO:2000344), and cell recognition (GO:0008037). Figure S2c-d shows the linkages of genes and biological concepts of the enriched GO terms as a network.

A total of 7 and $12 \mathrm{GO}$ terms with $P$-adjust $<0.05$ in biological process and molecular function terms, respectively, were significantly enriched in the liver of medaka by the inclusion of silkrose-BM in their diet (Figure S4a-b and Table S6), including cellular iron homeostasis (GO:0006879), Kupffer's vesicle development (GO:0070121), serine-type endopeptidase activity (GO:0004252), chemokine activity 
(GO:0008009), transmembrane signalling receptor activity (GO:0004888), molecular transducer activity (GO:0060089), organic acid binding (GO:0043177), and carboxylic acid binding (GO:0031406). The network plot of gene-gene linkages and biological concepts of enriched GO terms is shown in Figure S4c-d.

\section{KEGG enrichment results of DEGs}

To determine the signalling pathways of DEGs in each type of tissue sample, KEGG enrichment analysis was performed using the software package ClusterProfiler. DEGs from the comparison between $\mathrm{S}-\mathrm{N}$ and $\mathrm{C}-\mathrm{N}$ groups were mapped into the KEGG pathway database (https://www.kegg.jp/) and then differences were calculated using $P$-adjust $<0.05$ as the threshold of statistical significance. In the intestine, eight signalling pathways were significantly enriched, including cardiac muscle contraction (ola04260), calcium signalling pathway (ola04020), glycolysis/gluconeogenesis (ola00010), and glycosphingolipid biosynthesis (ola00604) (Figure S5a and Table S7). On the other hand, KEGG enrichment analysis of DEGs extracted from the liver of medaka showed that 16 signalling pathways were significantly enriched, including retinol metabolism (ola00830), steroid hormone biosynthesis (ola00140), mucin type O-glycan biosynthesis (ola00512), one carbon pool by folate (ola00670), glycolysis/gluconeogenesis (ola00010), insulin signalling pathway (ola04910), cytokine-cytokine receptor interaction (ola04060), intestinal immune network for IgA production (ola04672), and TLR signalling pathway (ola04620) (Figure S5b and Table s7). Figures S5c-d show the linkages between genes and biological concepts of the enriched KEGG pathways as a network.

\section{The change in TLR/NF-kB signalling pathway in response to dietary silkrose-BM}

Numerous genes in the liver of medaka involved in the TLR/ NF- $\kappa B$ signalling pathway were differentially expressed in response to dietary silkrose-AY. Down-regulated genes included TLR1, TLR9, MIP-1 $\beta$, FADD, AKT3b, IRF3, CD40, Map2k6, and FOSAb, while up-regulated genes included TLR5, TLR2/18, RAC1, PI3K, IRAK4, CTSL1, Map3k8/ JNK, Mapk10/JNK, IKK $\beta$, IL-1 $\beta$, IL12bb, PF2, Cxcl19/IL8, STAT1, and JUNE/AP1 (Table S8). Figure S9 shows the pathway linkages between all DEGs involved in TLR/NF- $\mathrm{BB}$ signalling pathway based on KEGG database annotation (https://www.kegg.jp/).

\section{The change in cytokine-cytokine receptor interactions in response to dietary silkrose-BM}

A series of CXC and CC motif of chemokine subfamilies and TNF families in cytokine-cytokine receptor interaction was found to be affected in the liver of medaka fed with silkrose-BM. KEGG enrichment of DEGs annotated in cytokine-cytokine receptor interactions showed an increase in the expression of several genes, such as IL2R $\beta$, IL-4R, IL-1 $\beta$, IL-1R2, IL12bb, IL-10R $\alpha$, IL-17R $\beta$, Ebi3/ IL-27, Cxcl19/IL-8, Cxcl14, Ackr4b/Ccr11, Ccl44/Ccl17, Csf2r $\beta$, Tnfrsf12a/Fn14, EPO, INH $\beta b$, GDF11, THPO, LIFR, OPGA/DCR3, GHR, TRAIL, BMP16, and LEPR (Table S8). Down-regulated genes included $\operatorname{Tgf} \beta 3, \operatorname{Tgf} \beta 5, \operatorname{Tgf} \beta \mathrm{r} 2$, IL-1R1, IL-12Rb1, IL-17c, IL-15Ra, IL-18R1, Ccl4/Ccl411/ Cc1412, Ccr7, Cxcr3, Cxcr4, Cxcr4b, Cxcl12, CD40, Clcf1, HVEM, MPL, Tnfr4/OX40, LIGHT, FASLG, and ACVR1 (Table S8). Figure S10 shows the hierarchical clustering of DEGs involved in cytokine-cytokine receptor interactions annotated in the KEGG pathway database (https://www. kegg.jp/).

\section{Identification of DEGs related to immunity}

We utilised a combination of GO annotation, KEGG pathway database, NCBI database, and manual literature searches to identify and categorise immune-related functional categories of DEGs identified from the comparison between the S-N and C-N groups. Six functional categories were observed (Table S8) to be potentially affected by dietary silkrose-BM in the intestine and liver of medaka, including: (1) TLR signalling pathway; (2) cytokine-cytokine receptor interaction; (3) complement cascade; (4) antimicrobial peptides; (5) junctional modification; and (6) other immune responses/inflammations. A detailed description of the functional roles and interactions of these genes is given in the Discussion, below.

\section{Validation of RNA-seq results by quantitative RT-PCR}

To verify our RNA-seq results, the relative expression of seven genes representing DEGs from the comparison between $\mathrm{S}-\mathrm{N}$ and $\mathrm{C}-\mathrm{N}$ groups was investigated using qRT-PCR. Spearman's correlation test was conducted to statistically compare the log2 fold-change from qRTPCR with the RNA-seq results. A significant positive correlation between the qRT-PCR and RNA-seq results was observed (Spearman's correlation coefficient $\rho=0.840$, $P<0.05)$, suggesting the reliability and accuracy of the RNAseq results for the gene expression analysis in this study (Figure S6).

\section{Discussion}

A growing interest has emerged in the use of naturallyderived immunostimulants as dietary supplements for aquatic animals. A broad range of immunostimulants, ranging from polysaccharides, animal and plants extracts, nutritional factors such as vitamins, and certain hormones and cytokines, are commonly added to the diet of aquatic animals to enhance the animals' resistance to pathogens and improve the animals' immunity, especially the nonspecific/innate immune system (Doñate et al., 2010; 
Sakai, 1999; Vallejos-Vidal et al., 2016). Recently, we have purified several novel acidic polysaccharides, from melon fly larvae (dipterose-BC; B. cucurbitae) (Ohta et al., 2014), black soldier fly larvae (dipterose-BSF; H. illucens) (Ali et al., 2019), Japanese oak silkmoth pupae (silkrose-AY; A. yamamai) (Ohta et al., 2015), and silkmoth pupae (silkrose-BM; B. mori) (Ali et al., 2018), which can activate mammalian innate immunity in mouse RAW264.7 macrophages via the TLR/NF- $\mathrm{kB}$ pathway, indicating their potential as immunostimulatory substances for the aquaculture industry. Assuming that these insect-derived polysaccharides could be practically used in the aquaculture industry, B. mori pupae have a unique advantage in terms of availability, as they are a by-product derived from silk production (Rahimnejad et al., 2019), suggesting a possible synergy between the aquaculture and sericulture industries in the future. As reported by Ping and Jie (2011), the total annual yield of silkworm cocoon in China reached approximately 575,300 tons in 2009 . In our previous study, we found that dietary silkrose-AY, an insect-derived polysaccharide from Japanese oak silkmoth (A. yamamai), can significantly improve the survival and decreased the number of bacteria in the kidney of Japanese medaka (O. latipes) after artificially infected with $E$. tarda (Ali et al., 2021). It is noteworthy that despite Japanese oak silkmoth (A. yamamai) and silkmoth (B. mori) are taxonomically related to each other, their derived polysaccharides are relatively different in terms of structural characterisations and biological activity. There were noticeable differences in the molecular weight and monosaccharide composition between silkrose-AY and silkrose-BM (Ohta et al., 2016; Ali et al., 2018). In addition, in vitro analysis using mouse macrophage RAW264.7 cell line indicates that silkroseBM is recognised by both TLR2 and TLR4, in contrast to silkrose-AY that exhibited recognition by TLR4 but not TLR2 Ohta et al., 2015). As the efficacy of silkrose-BM in teleosts remains largely unclear, here we utilised this novel immunostimulatory polysaccharide derived from the silkmoth (B. mori) in the diet of Japanese medaka (O. latipes). We then analysed changes in the transcriptome profile using RNA-seq technology.

In the present study, dietary inclusion of silkrose-BM at 10,100 , or $1000 \mathrm{ng} / \mathrm{g}$ in medaka resulted in a significant improvement in the survival of the fish following artificial infection with $E$. tarda. A similar situation was observed in our previous study, in which the dietary inclusion of silkrose-BM in two penaeid prawns (Litopenaeus vannamei and Marsupenaeus japonicus) significantly decreased the mortality of the prawns after they were infected with Vibrio penaecida (Ali et al., 2018). We also found that dietary silkrose-AY, an immunostimulatory polysaccharide derived from A. yamamai, significantly enhanced the survival of fish and decreased the concentration of bacteria in the kidneys of medaka following $E$. tarda infection (Ali et al., 2021). Chitin, a major constituent of insect exoskeletons, is another polysaccharide that is commonly associated with the immunostimulatory effect of insect-containing diets (Lee et al., 2008). Dietary inclusion of at least 1\% chitin was reported to effectively prevent diseases in species including common carp (Cyprinus carpio) (Gopalakannan and Arul, 2006), longtooth grouper (Epinephelus bruneus) (Harikrishnan et al., 2012a,b), Mrigal carp (Cirrhinus mrigala) (Shanthi Mari et al., 2014), and rohu (Labeo rohita) (Kumar et al., 2019). However, it is noteworthy that silkrose$\mathrm{BM}$ and chitin have different structural characterisations, such as molecular weight and monosaccharide composition. In addition, compared with the use of chitin as an immunostimulant, an important aspect of silkrose-BM is that it is effective at a very low dosage (around $10 \mathrm{ng} / \mathrm{g}$ ). Taken together, our current results indicate that silkrose-BM could be beneficial to the aquaculture industry as a functional feed ingredient for protection against bacterial infections.

In the present study, RNA-seq technology was used to reveal changes in the transcriptional profile of DEGs in the intestine and liver of medaka resulting from dietary silkroseBM. Various functional categories related to the immune system were identified as being potentially associated with the dietary inclusion of silkrose-BM, including TLR pathway, cytokine-cytokine receptor interactions, complement cascade, macrophage activation, antimicrobial peptides, and junctional modification. In addition, several non-immune-related functional categories were observed in the intestine and liver of medaka. Below, we describe several key constituents of these functional categories and their potential function with regard to medaka immune system responses to dietary silkrose-BM.

\section{TLR/nuclear factor KB (NF-kB) signalling pathway}

It is a widely accepted concept that the activation of innate immunity is initiated by the recognition of foreign surface components, known as pathogen-associated molecular patterns (PAMPs), through pathogen-recognition receptors (PRRs) such as TLRs (Akira et al., 2006; Kawai and Akira, 2010). In this study, functional enrichment analysis revealed that the TLR signalling pathway in the liver of medaka was significantly affected by dietary silkrose-BM. PAMPrecognising TLRs, such as TLR1 and TLR9, were found to be down-regulated, while TLR2/18 and TLR5 were found to be up-regulated. This observation was analogous with those of our previous studies, in which insect-derived polysaccharides, including dipterose-BC, dipterose-BSF, and silkrose-AY, were found to be associated with TLR2 and TLR4 and induce immunostimulatory activities in mammalian RAW264.7 macrophages (Ali et al., 2019; Ohta et al., 2014, 2015). Our findings thus suggest that silkrose-BM acts as a PAMP for teleosts. Figure S9 shows that the stimulation of PRRs by silkrose-BM triggered several downstream signalling events, including both MYD88-dependent and -independent pathways, leading 
to the production of inflammatory cytokines such as IL$1 \beta$, IL-12, and IL-8. These phenomena are similar to what we found before in mammalian RAW264.7 macrophages treated with insect-derived polysaccharides, including dipterose-BC, dipterose-BSF, silkrose-AY, and silkrose-BM (Ali et al., 2018, 2019; Ohta et al., 2014, 2015). Ferreira et al. (2015) reported that the immunostimulatory activity of polysaccharides is closely related to their structural characterisation being similar to the molecular patterns found in pathogens that are generally absent from the host. Therefore, we hypothesise that silkrose-BM may serve as PAMP and utilise TLRs pathway to stimulate innate immune responses by mimicking the early phase of bacterial infection, leading to better preparation of host immune system against pathogen invasion.

\section{Changes in cytokine-cytokine receptor interactions}

Generally, inflammatory responses are tightly regulated by the interaction between cytokine components; the activation of inflammatory responses is aimed at enhancing the antimicrobial capacity of immune cells, facilitating pathogen clearance, and assisting tissue repair mechanisms (Grayfer and Belosevic, 2012; Morimoto et al., 2021). In the current study, we found various cytokines and their putative receptors were significantly affected in the liver of medaka fed with dietary silkrose-BM. Chemokines (Cxcl19/IL-8, Cxcl14, Ackr4b/Ccr11, Ccl44/Ccl17, Csf2r $\beta$, Ccl4/Ccl411/ Ccl412, Ccr7, Cxcr3, Cxcr4, Cxcr4b, Cxcl12, CD40, and Clcf1), interleukins (IL2R $\beta$, IL-4R, IL-1 $\beta$, IL-1R2, IL12bb, IL-10R $\alpha$, IL-17R $\beta$, Ebi3/IL-27, IL-1R1, IL-12Rb1, IL-17c, IL-15Ra, and IL-18R1), and tumour necrosis factors (TNF; Tnfrsf12a/Fn14, CD40, HVEM, Tnfr4/OX40, and LIGHT) were among the cytokine-cytokine receptor interactions that were found to be up- and down-regulated due to the dietary inclusion of silkrose-BM (Figure S10; Table S8). This is consistent with the findings of a previous report that showed IL- $1 \beta$ and IL-12 up-regulation in response to lipopolysaccharide, a PAMP-containing polysaccharide, in rainbow trout (Oncorhynchus mykiss) and common carp (C. carpio) (Arts et al., 2010; Wang et al., 2009). Naturally-derived polysaccharides, such as those from Chlorophytum borivilianum, Astragalus spp., and Ficus carica, were also found to stimulate IL-1 $\beta$ expression in $L$. rohita (Giri et al., 2015), C. carpio (Yuan et al., 2008), and Ctenopharyngodon idella (Yang et al., 2015), respectively. Moreover, the anti-inflammatory cytokines observed to be down-regulated in the present study, such as Tgf $\beta 3$ and $\operatorname{Tgf} \beta 5$, were also observed in L. rohita fed with $C$. borivilianum-derived polysaccharide (Giri et al., 2015). Thus, because the functional categories of cytokinecytokine receptor interactions were significantly enriched in the liver of medaka fed on silkrose-BM, we speculate that blood coming from the gastrointestinal tract might be enriched in antigens, in this case, silkrose-BM, and immune cells in the liver recognised this via TLRs, thus activating various proinflammatory cytokines and enhancing the host immune system against bacterial infection.

\section{Complement cascade}

The current study showed numerous genes related to the complement cascade were altered by dietary silkroseBM in both the liver and intestine of medaka (Table S8). Complement activation during pathogen infection provides a broad range of defensive functions in teleosts (Boshra et al., 2006; Holland and Lambris, 2002). The up-regulation of the complement components C1q, C3, C3-1, C3b, C7b, and $\mathrm{C} 8 \mathrm{~b}$, and the complement factors $\mathrm{CfH}$ and $\mathrm{CfB}$ in the current study is similar to what we found previously in medaka fed on silkrose-AY, an insect-derived polysaccharide from $A$. yamamai (Ali et al., 2021). The activation of C3, C3-1, and $\mathrm{C} 3 \mathrm{~b}$, pivotal components in the complement cascade, as well as $\mathrm{C} 7 \mathrm{~b}$ and $\mathrm{C} 8 \mathrm{~b}$, complement components responsible for the formation of the membrane attack complex, in the current study, indicates that silkrose-BM might provide the host with bacterial resistance by utilising the bactericidal properties of the complement system (Boshra et al., 2006; Holland and Lambris, 2002). The ability of the complement cascade to enhance the phagocytic activity of macrophages and monocytes is analogous with our previous finding of increasing phagocytic activity of red sea bream peritoneal leukocytes after the fish were fed with an insect-containing diet (Ido et al., 2015). Lipopolysaccharide (Sunyer and Tort, 1995) and naturally-derived polysaccharides, such as those from F. carica (Yang et al., 2015), Allium mongolicum Regel (Li et al., 2019), and C. borivilianum (Giri et al., 2015), have also been found to be associated with teleost complement system activation. Taken together, our results indicate that the complement system might play a crucial role in the immunostimulatory properties of silkrose-BM that enhance innate immunity in teleosts.

\section{Antimicrobial peptides and other immune-related genes}

A broad range of antimicrobial peptides is present in the liver and gastrointestinal tract (Katzenback, 2015; Peatman et al., 2015). In the current study, we observed that silkrose-BM elevated the expression of bactericidal permeability-increasing protein, hepcidin, and liverexpressed antimicrobial peptide 2 in the liver and intestine of medaka (Table S8). These molecules help to prevent bacterial infection by directly killing invading bacteria via cell lysis, enhancing phagocytosis, and modulating innate immune responses (Li et al., 2013, 2015; Sun and Sun, 2016). Arginase 2, a molecule involved in the alternative activation of macrophages (Joerink et al., 2006), was also found to be up-regulated by silkrose-BM (Table S8), suggesting that silkrose-BM could trigger both classically-activated macrophages via IL-1 $\beta$ activation and alternatively-activated macrophages via arginase 2 activation. More intriguingly, we observed increased expression of immunoresponsive 
gene 1 in the intestine of medaka fed on silkrose-BM. This molecule can effectively inhibit the growth of Salmonella enterica and Mycobacterium tuberculosis by catalysing itaconic acid production, an organic compound that inhibits isocitrate lyase (part of a pathway essential to bacterial growth) (Michelucci et al., 2013). Based on these results, silkrose-BM seems to be capable of altering various immune responses to enhance a host's defence mechanisms against invasive pathogens.

\section{Non-immune-related genes}

Aside from immune-related genes, our transcriptome profiling also suggested the enrichment of several nonimmune-related functional categories in both the intestine and liver of medaka following dietary inclusion of silkroseBM (Figures S2, S3, and S4). These non-immune categories included muscle contraction (GO:0006936), insulin signalling pathway (ola04910), and actomyosin structure organisation (GO:0031032), which could be categorised as growth-related functional categories, while fertilisation/ reproduction (GO:0009566; GO:0000003) and positive regulation of acrosome (GO:2000344) were representative of reproduction-related categories. Recent studies in Salmo salar have shown that IL-1 $\beta$ can induce genes related to muscle metabolism and growth, similar to our current results, suggesting additional roles for IL-1 $\beta$ besides immune regulation (Pooley et al., 2013). To the best of our knowledge, there have yet to be any studies investigating the effect of insect-derived polysaccharides on reproduction in teleosts. Therefore, further investigation in this regard is needed to understand the underlying mechanisms of these phenomena.

\section{Conclusions}

In summary, we have demonstrated that dietary silkrose$\mathrm{BM}$, an insect-derived polysaccharide obtained from B. mori, effectively prevents edwardsiellosis in Japanese medaka. Furthermore, transcriptome profiling of the intestine and liver of medaka indicated that silkrose-BM might stimulate various host immune responses to help prevent bacterial infection, providing comprehensive information about the molecular mechanism underlying the modulatory effect of silkrose-BM as an immunostimulant in teleosts. The current study further suggests the application of insects not only as a viable source of animal protein but also as a promising alternative for disease control in the aquaculture industry. Although a transcriptional analysis by RNAseq was conducted in this study, transcriptional profiling itself does not take into account post-transcriptional and post-translational regulation of protein expression; thus, further studies using other 'omics' technology, such as proteomics, could help resolve this limitation. Moreover, further investigation of the efficacy of silkrose-BM in commercial fish species is needed to elucidate the optimal dose for practical application as well as to explore any long-term effects, not only in terms of the immune system but also other aspects, such as growth and reproduction.

\section{Ethics approval}

Animal experiments were carried out following the guidelines of Ehime University. The study protocol was accepted by the Institutional Animal Care and Use Committee (IACUC) of Ehime University (Permit Number: 3908). 2-phenoxyethanol was used while performing all body measurements and surgical manipulations at concentrations of 0.01 and $0.2 \%$, respectively. All efforts were made to minimise suffering.

\section{Supplementary material}

Supplementary material can be found online at https://doi. org/10.3920/JIFF2021.0108.

Table S1. Composition of the basal diet for medaka (Oryzias latipes).

Table S2. Primer used for quantitative real-time RT-PCR analysis.

Table S3. The RNA-seq summary results of raw reads, clean reads, and values of Q20 and Q30.

Table S4. The RNA-seq summary table of clean reads and mapping results.

Table S5. Result of significantly enriched gene ontologies (adjusted $P$-value $<0.05$ ) in the intestine of medaka calculated by ClusterProfiler (ver. 3.18.1) using DEGs from the control $(\mathrm{C}-\mathrm{N})$ and silkrose-BM (S-N) groups comparison.

Table S6. Result of significantly enriched gene ontologies (adjusted $P$-value $<0.05)$ in the liver of medaka calculated by ClusterProfiler (ver. 3.18.1) using DEGs from the control $(\mathrm{C}-\mathrm{N})$ and silkrose-BM (S-N) groups comparison.

Table S7. Result of significantly enriched Kyoto Encyclopaedia of Genes and Genomes (KEGG) pathway (adjusted $P$-value $<0.05$ ) calculated by ClusterProfiler (ver. 3.18.1) using DEGs from the comparison between control $(\mathrm{C}-\mathrm{N})$ and silkroseBM (S-N).

Table S8. List of differentially expressed immune-related genes ( $\mid \log 2$ fold-change $\mid \geq 1$ and $P$-value $<0.05)$ and their expression ratio in the liver and intestine of medaka.

Figure S1. Transcriptional profiling analysis of the intestine and liver of medaka. 
Figure S2. Gene ontology (GO) enrichment analysis in the intestine of medaka using DEGs resulted from the comparison between $\mathrm{S}-\mathrm{N}$ and $\mathrm{C}-\mathrm{N}$.

Figure S3. REVIGO analysis results for GO terms of biological processes in the intestine.

Figure S4. Gene ontology (GO) enrichment analysis in the liver of medaka using DEGs resulted from the comparison between silkrose-BM (S-N) and control (C-N) groups.

Figure S5. Kyoto Encyclopaedia of Genes and Genomes (KEGG) pathway enrichment analysis.

Figure S6. Validation of RNA-seq results by quantitative qRT-PCR.

Figure S7. Standard curves were generated from a serial 4-fold dilution of a control sample for each gene-specific primer used in the qRT-PCR analysis.

Figure S8. $\beta$-actin gene expression across all treatment conditions.

Figure S9. The change of Toll-like receptor signalling pathway in the liver of medaka in response to dietary silkrose-Bombyx mori.

Figure S10. The change of cytokine-cytokine receptor interaction in the liver of medaka in response to dietary silkrose-Bombyx mori.

\section{Acknowledgements}

We are grateful to the staff of the Laboratory of Fish Reproductive Physiology, the Graduate School of Agriculture, Ehime University for their assistance in this study. We also thank the United Silk Co., Ltd. and Ainan Liberacio Co. Ltd. for providing us with silkworm pupae. This work was supported by the Japan Science and Technology Agency and JSPS Kakenhi (18H03960).

\section{Conflict of interest}

The authors declare that they have no competing financial interests.

\section{References}

Akira, S., Uematsu, S. and Takeuchi, O., 2006. Pathogen recognition and innate immunity. Cell 124: 783-801. https://doi.org/10.1016/j. cell.2006.02.015
Ali, M.F.Z., Kameda, K., Kondo, F., Iwai, T., Kurniawan, R.A., Ohta, T., Ido, A., Takahashi, T., Miura, C. and Miura, T., 2021. Effects of dietary silkrose of Antheraea yamamai on gene expression profiling and disease resistance to Edwardsiella tarda in Japanese medaka (Oryzias latipes). Fish and Shellfish Immunology 114: 207-217. https://doi.org/10.1016/j.fsi.2021.05.001

Ali, M.F.Z., Ohta, T., Ido, A., Miura, C. and Miura, T., 2019. The dipterose of black soldier fly (Hermetia illucens) induces innate immune response through toll-like receptor pathway in mouse macrophage RAW264.7 cells. Biomolecules 9: 677. https://doi. org/10.3390/biom9110677

Ali, M.F.Z., Yasin, I.A., Ohta, T., Hashizume, A., Ido, A., Takahashi, T., Miura, C. and Miura, T., 2018. The silkrose of Bombyx mori effectively prevents vibriosis in penaeid prawns via the activation of innate immunity. Scientific Reports 8: 8836. https://doi.org/10.1038/ s41598-018-27241-3

Arts, J.A., Tijhaar, E.J., Chadzinska, M., Savelkoul, H.F. and VerburgVan Kemenade, B.M., 2010. Functional analysis of carp interferon- $\gamma$ : evolutionary conservation of classical phagocyte activation. Fish and Shellfish Immunology 29: 793-802. https://doi.org/10.1016/j. fsi.2010.07.010

Bebak, J. and Wagner, B., 2012. Use of vaccination against enteric septicemia of catfish and columnaris disease by the U.S. Catfish Industry. Journal of Aquatic Animal Health 24: 30-36. https://doi. org/10.1080/08997659.2012.667048

Boshra, H., Li, J. and Sunyer, J.O., 2006. Recent advances on the complement system of teleost fish. Fish and Shellfish Immunology 20: 239-262. https://doi.org/10.1016/j.fsi.2005.04.004

Cabello, F.C., 2006. Heavy use of prophylactic antibiotics in aquaculture: a growing problem for human and animal health and for the environment. Environmental Microbiology 8: 1137-1144. https://doi.org/10.1111/j.1462-2920.2006.01054.x

Castro, N., Toranzo, A.E., Núñez, S. and Magariños, B., 2008. Development of an effective Edwardsiella tarda vaccine for cultured turbot (Scophthalmus maximus). Fish and Shellfish Immunology 25: 208-212. https://doi.org/10.1016/j.fsi.2008.05.008

Chaklader, M.R., Siddik, M.A.B., Fotedar, R. and Howieson, J., 2019. Insect larvae, Hermetia illucens in poultry by-product meal for barramundi, Lates calcarifer modulates histomorphology, immunity and resistance to Vibrio harveyi. Scientific Reports 9: 16703. https:// doi.org/10.1038/s41598-019-53018-3

Chernysh, S., Kim, S.I., Bekker, G., Pleskach, V.A., Filatova, N.A., Anikin, V.B., Platonov, V.G. and Bulet, P., 2002. Antiviral and antitumor peptides from insects. Proceedings of the National Academy of Sciences of the USA 99: 12628-12632. https://doi. org/10.1073/pnas.192301899

Clavijo, A.M., Conroy, G., Conroy, D.A., Santander, J. and Aponte, F., 2002. First report of Edwardsiella tarda from tilapias in Venezuela. Bulletin of the European Association of Fish Pathologists 22: 280282.

Doñate, C., Balasch, J.C., Callol, A., Bobe, J., Tort, L. and MacKenzie, S., 2010. The effects of immunostimulation through dietary manipulation in the rainbow trout: evaluation of mucosal immunity. Marine Biotechnology 12: 88-99. https://doi.org/10.1007/s10126009-9203-4 
Dubois, M., Gilles, K.A., Hamilton, J.K., Rebers, P.A. and Smith, F., 1956. Colorimetric method for determination of sugars and related substances. Analytical Chemistry 28: 350-356. https://doi. org/10.1021/ac60111a017

Ferreira, S.S., Passos, C.P., Madureira, P., Vilanova, M. and Coimbra, M.A., 2015. Structure - function relationships of immunostimulatory polysaccharides: a review. Carbohydrate Polymers 132: 378-396. https://doi.org/10.1016/j.carbpol.2015.05.079

Gasco, L., Finke, M. and Van Huis, A., 2018. Can diets containing insects promote animal health? Journal of Insects as Food and Feed 4: 1-4. https://doi.org/10.3920/JIFF2018.x001

Giri, S.S., Sen, S.S., Chi, C., Kim, H.J., Yun, S., Park, S.C. and Sukumaran, V., 2015. Chlorophytum borivilianum polysaccharide fraction provokes the immune function and disease resistance of Labeo rohita against Aeromonas hydrophila. Journal of Immunology Research, Article ID: 256510. https://doi.org/10.1155/2015/256510

Gopalakannan, A. and Arul, V., 2006. Immunomodulatory effects of dietary intake of chitin, chitosan and levamisole on the immune system of Cyprinus carpio and control of Aeromonas hydrophila infection in ponds. Aquaculture 255: 179-187. https://doi. org/10.1016/j.aquaculture.2006.01.012

Grayfer, L. and Belosevic, M., 2012. Cytokine regulation of teleost inflammatory responses. In: Turker, H. (ed.) New advances and contributions to fish biology. IntechOpen, London, UK, pp. 59-96.

Harikrishnan, R., Kim, J.S., Balasundaram, C. and Heo, M.S., 2012. Dietary supplementation with chitin and chitosan on haematology and innate immune response in Epinephelus bruneus against Philasterides dicentrarchi. Experimental Parasitology 131: 116124. https://doi.org/10.1016/j.exppara.2012.03.020

Harikrishnan, R., Kim, J.S., Balasundaram, C. and Heo, M.S., 2012. Immunomodulatory effects of chitin and chitosan enriched diets in Epinephelus bruneus against Vibrio alginolyticus infection. Aquaculture 326-329: 46-52. https://doi.org/10.1016/j. aquaculture.2011.11.034

Henry, M.A., Gai, F., Enes, P., Peréz-Jiménez, A. and Gasco L., 2018. Effect of partial dietary replacement of fishmeal by yellow mealworm (Tenebrio molitor) larvae meal on the innate immune response and intestinal antioxidant enzymes of rainbow trout (Oncorhynchus mykiss). Fish and Shellfish Immunology 83: 308-313. https://doi. org/10.1016/j.fsi.2018.09.040

Holland, M.C.H. and Lambris, J.D., 2002. The complement system in teleosts. Fish and Shellfish Immunology 12: 399-420. https://doi. org/10.1006/fsim.2001.0408

Ido, A., Iwai, T., Ito, K., Ohta, T., Mizushige, T., Kishida, T., Miura, C. and Miura, T., 2015. Dietary effects of housefly (Musca domestica) (Diptera: Muscidae) pupae on the growth performance and the resistance against bacterial pathogen in red sea bream (Pagrus major) (Perciformes: Sparidae). Applied Entomology and Zoology 50: 213-221. https://doi.org/10.1007/s13355-015-0325-Z

Ido, A., Hashizume, A., Ohta, T., Takahashi, T., Miura, C. and Miura, T., 2019. Replacement of fish meal by defatted yellow mealworm (Tenebrio molitor) larvae in diet improves growth performance and disease resistance in red seabream (Pagrus major). Animals 9: 100. https://doi.org/10.3390/ani9030100
Joerink, M., Savelkoul, H.F. and Wiegertjes, G.F., 2006. Evolutionary conservation of alternative activation of macrophages: structural and functional characterization of arginase 1 and 2 in carp (Cyprinus carpio L.). Molecular Immunology 43: 1116-1128. https://doi. org/10.1016/j.molimm.2005.07.022

Katzenback, B.A., 2015. Antimicrobial peptides as mediators of innate immunity in teleosts. Biology 4: 607-639. https://doi.org/10.3390/ biology 4040607

Kawai, T. and Akira, S., 2010. The role of pattern-recognition receptors in innate immunity: update on toll-like receptors. Nature Immunology 11: 373-384. https://doi.org/10.1038/ni.1863

Kim, D., Paggi, J.M., Park, C., Bennett, C. and Salzberg, S.L., 2019. Graph-based genome alignment and genotyping with HISAT2 and HISAT-genotype. Nature Biotechnology 37(8): 907-915. https://doi. org/10.1038/s41587-019-0201-4.

Kumar, R., Kaur, N. and Kamilya, D., 2019. Chitin modulates immunity and resistance of Labeo rohita (Hamilton, 1822) against gill monogeneans. Aquaculture 498: 522-527. https://doi.org/10.1016/j. aquaculture.2018.09.013

Lee, C.G., Da Silva, C.A., Lee, J.Y., Hartl, D. and Elias, J.A., 2008. Chitin regulation of immune responses: an old molecule with new roles. Current Opinion in Immunology 20: 684-689. https://doi. org/10.1016/j.coi.2008.10.002

Li, H., Handsaker, B., Wysoker, A., Fennell, T., Ruan, J., Homer, N., Marth, G., Abecasis, G., Durbin, R. and 1000 Genome Project Data Processing Subgroup, 2009. The sequence alignment/map format and SAMtools. Bioinformatics 25(16): 2078-2079. https:// doi.org/10.1093/bioinformatics/btp352

Li, H., Zhang, F., Guo, H., Zhu, Y., Yuan, J., Yang, G. and An, L., 2013. Molecular characterization of hepcidin gene in common carp (Cyprinus carpio L.) and its expression pattern responding to bacterial challenge. Fish and Shellfish Immunology 35: 1030-1038. https://doi.org/10.1016/j.fsi.2013.07.001

Li, H.X., Lu, X.J., Li, C.H. and Chen, J., 2015. Molecular characterization of the liver-expressed antimicrobial peptide 2 (LEAP-2) in a teleost fish, Plecoglossus altivelis: antimicrobial activity and molecular mechanism. Molecular Immunology 65: 406-415. https://doi. org/10.1016/j.molimm.2015.02.022

Li, M.Y., Zhu, X.M., Niu, X.T., Chen, X.M., Tian, J.X., Kong, Y.D., Zhang, D.M., Zhao, L. and Wang, G.Q., 2019. Effects of dietary Allium mongolicum Regel polysaccharide on growth, lipopolysaccharideinduced antioxidant responses and immune responses in Channa argus. Molecular Biology Reports 46: 2221-2230. https://doi. org/10.1007/s11033-019-04677-y

Liao, Y., Smyth, G.K. and Shi, W., 2014. featureCounts: an efficient general purpose program for assigning sequence reads to genomic features. Bioinformatics 30(7): 923-930. https://doi.org/10.1093/ bioinformatics/btt656

Livak, K.J. and Schmittgen, T.D., 2001. Analysis of relative gene expression data using real-time quantitative PCR and the 2(-Delta Delta C(T)) method. Methods 25: 402-408. https://doi.org/10.1006/ meth.2001.1262

Makkar, H.P.S., Tran, G., Heuzé, V. and Ankers, P., 2014. Stateof-the-art on use of insects as animal feed. Animal Feed Science and Technology 197: 1-33. https://doi.org/10.1016/j. anifeedsci.2014.07.008 
Martin, M., 2011. Cutadapt removes adapter sequences from highthroughput sequencing reads. EMBnet.journal 17(1): 10-12. https:// doi.org/10.14806/ej.17.1.200

Martin, S.A.M., Dehler, C.E. and Król, E., 2016. Transcriptomic responses in the fish intestine. Developmental and Comparative Immunology 64: 103-117. https://doi.org/10.1016/j.dci.2016.03.014

Matsuyama, T., Kamaishi, T., Ooseko, N., Kurohara, K. and Iida T., 2005. Pathogenicity of motile and non-motile Edwardsiella tarda to some marine fish. Fish Pathology 40: 133-135. https://doi. org/10.3147/JSFP.40.133

Meyer, F.P. and Bullock, G.L., 1973. Edwardsiella tarda, a new pathogen of channel catfish (Ictalurus punctatus). Applied Microbiology 25: 155-156. https://doi.org/10.1128/am.25.1.155-156.1973

Michelucci, A., Cordes, T., Ghelfi, J., Pailot, A., Reiling, N., Goldmann, O., Binz, T., Wegner, A., Tallam, A., Rausell, A., Buttini, M., Linster, C.L., Medina, E., Balling, R. and Hiller, K., 2013. Immune-responsive gene 1 protein links metabolism to immunity by catalyzing itaconic acid production. Proceedings of the National Academy of Sciences of the USA 110: 7820-7825. https://doi.org/10.1073/pnas.1218599110

Mohanty, B.R. and Sahoo, P.K., 2007. Edwardsiellosis in fish: a brief review. Journal of Biosciences 32: 1331-1344. https://doi. org/10.1007/s12038-007-0125-x

Morimoto, N., Kono, T., Sakai, M. and Hikima, J.I., 2021. Inflammasomes in teleosts: structures and mechanisms that induce pyroptosis during bacterial infection. International Journal of Molecular Sciences 22: 4389. https://doi.org/10.3390/ijms22094389

Motte, C., Rios, A., Lefebvre, T., Do, H., Henry, M. and Jintasataporn, O., 2019. Replacing fish meal with defatted insect meal (Yellow mealworm Tenebrio molitor) improves the growth and immunity of pacific white shrimp (Litopenaeus vannamei). Animals 9: 258. https://doi.org/10.3390/ani9050258

Ohta, T., Ido, A., Kusano, K., Miura, C. and Miura, T., 2014. A novel polysaccharide in insects activates the innate immune system in mouse macrophage RAW264 cells. PLoS ONE 9: e114823. https:// doi.org/10.1371/journal.pone.0114823

Ohta, T., Kusano, K., Ido, A., Miura, C. and Miura, T., 2015. Silkrose: a novel acidic polysaccharide from the silkmoth that can stimulate the innate immune response. Carbohydrate Polymers 136: 995-1001. https://doi.org/10.1016/j.carbpol.2015.09.070

Padrós, F., Zarza, C., Dopazo, L., Cuadrado, M. and Crespo, S., 2006. Pathology of Edwardsiella tarda infection in turbot, Scophthalmus maximus (L.). Journal of Fish Diseases 29: 87-94. https://doi. org/10.1111/j.1365-2761.2006.00685.x

Park, S.B, Aoki, T. and Jung, T.S., 2012. Pathogenesis of and strategies for preventing Edwardsiella tarda infection in fish. Veterinary Research 43: 67. https://doi.org/10.1186/1297-9716-43-67

Peatman, E., Lange, M., Zhao, H. and Beck, B.H., 2015. Physiology and immunology of mucosal barriers in catfish (Ictalurus spp.). Tissue Barriers 3: e1068907. https://doi.org/10.1080/21688370.2 015.1068907

Ping, K.G. and Jie, G.X., 2011. Overview of silkworm pathology in China. African Journal of Biotechnology 10: 18046-18056. https:// doi.org/10.5897/AJB10.263
Pooley, N.J., Tacchi, L., Secombes, C.J. amd Martin, S.A., 2013. Inflammatory responses in primary muscle cell cultures in Atlantic salmon (Salmo salar). BMC Genomics 14: 747. https:// doi.org/10.1186/1471-2164-14-747

Rahimnejad, S., Hu, S., Song, K., Wang, L., Lu, K., Wu, R. and Zhang, C., 2019. Replacement of fish meal with defatted silkworm (Bombyx mori L.) pupae meal in diets for Pacific white shrimp (Litopenaeus vannamei). Aquaculture 510: 150-159. https://doi.org/10.1016/j. aquaculture.2019.05.054

Ratcliffe, N., Azambuja, P. and Mello., C.B., 2014. Recent advances in developing insect natural products as potential modern day medicines. Evidence-Based Complementary and Alternative Medicine, Article ID 904958. https://doi.org/10.1155/2014/904.958

R Core Team, 2020. R: a language and environment for statistical computing. R Foundation for Statistical Computing, Vienna, Austria. Available at: https://www.R-project.org/.

Sakai, M., 1999. Current research status of fish immunostimulants. Aquaculture 172: 63-92. https://doi.org/10.1016/S00448486(98)00436-0

Salinas, I. and Magadán, S., 2017. Omics in fish mucosal immunity. Developmental and Comparative Immunology 75: 99-108. https:// doi.org/10.1016/j.dci.2017.02.010

Shanthi Mari, L.S., Jagruthi, C., Anbazahan, S.M., Yogeshwari, G., Thirumurugan, R., Arockiaraj, J., Mariappan, P., Balasundaram, C. and Harikrishnan, R., 2014. Protective effect of chitin and chitosan enriched diets on immunity and disease resistance in Cirrhina mrigala against Aphanomyces invadans. Fish and Shellfish Immunology 39: 378-385. https://doi.org/10.1016/j.fsi.2014.05.027

Sun, J., Nishiyama, T., Shimizu, K. and Kadota, K., 2013. TCC: an R package for comparing tag count data with robust normalization strategies. BMC Bioinformatics 14: 219.

Sun, Y.Y. and Sun, L., 2016. A teleost bactericidal permeabilityincreasing protein kills gram-negative bacteria, modulates innate immune response, and enhances resistance against bacterial and viral infection. PLoS ONE 11: e0154045. https://doi.org/10.1371/ journal.pone.0154045

Sunyer, J.O. and Tort, L., 1995. Natural hemolytic and bactericidal activities of sea bream Sparus aurata serum are effected by the alternative complement pathway. Veterinary Immunology and Immunopathology 45: 333-345. https://doi.org/10.1016/01652427(94)05430-z

Supek, F., Bošnjak, M., Škunca, N. and Šmuc, T., 2011. REVIGO summarizes and visualizes long lists of gene ontology terms. PLoS ONE 6: e21800. https://doi.org/10.1371/journal.pone.0021800

Vallejos-Vidal, E., Reyes-López, F., Teles, M. and MacKenzie, S., 2016. The response of fish to immunostimulant diets. Fish and Shellfish Immunology 56: 34-69. https://doi.org/10.1016/j.fsi.2016.06.028

Van Huis, A., 2021. Prospects of insects as food and feed. Organic Agriculture 11: 301-308. https://doi.org/10.1007/s13165-02000290-7

Wang, T., Bird, S., Koussounadis, A., Holland, J.W., Carrington, A., Zou, J. and Secombes, C.J., 2009. Identification of a novel IL-1 cytokine family member in teleost fish. The Journal of Immunology 183: 962-974. https://doi.org/10.4049/jimmunol.0802953 
Xiao, X., Jin, P., Zheng, L., Cai, M., Yu, Z., Yu, J. and Zhang, J., 2018. Effects of black soldier fly (Hermetia illucens) larvae meal protein as a fishmeal replacement on the growth and immune index of yellow catfish (Pelteobagrus fulvidraco). Aquaculture Research 49: 1569-1577. https://doi.org/10.1111/are.13611

Yang, X., Guo, J.L., Ye, J.Y., Zhang, Y.X. and Wang, W., 2015. The effects of Ficus carica polysaccharide on immune response and expression of some immune-related genes in grass carp, Ctenopharyngodon idella. Fish and Shellfish Immunology 42: 132-137. https://doi. org/10.1016/j.fsi.2014.10.037
Ye, H., Lin, Q. and Luo, H., 2018. Applications of transcriptomics and proteomics in understanding fish immunity. Fish and Shellfish Immunology 77: 319-327. https://doi.org/10.1016/j.fsi.2018.03.046

Yu, G., Wang, L.G., Han, Y. and He, Q.Y., 2012. clusterProfiler: an R package for comparing biological themes among gene clusters. OMICS 16(5): 284-287. https://doi.org/10.1089/omi.2011.0118

Yuan, C., Pan, X., Gong, Y., Xia, A., Wu, G., Tang, J. and Han, X., 2008. Effects of Astragalus polysaccharides (APS) on the expression of immune response genes in head kidney, gill and spleen of the common carp, Cyprinus carpio L. International Immunopharmacology 8: 51-58. https://doi.org/10.1016/j. intimp.2007.10.009 
